No. 1095

Optimal Call Admission Control for Voice Traffic in Cellular Mobile Communication Networks

by

Minoru Ohmikawa, Hideaki Takagi, and Sang-Yong Kim

September 2004 


\title{
Optimal Call Admission Control for Voice Traffic in Cellular Mobile Communication Networks
}

\author{
Minoru Ohmikawa \\ Doctoral Program in Policy and Planning Sciences \\ University of Tsukuba \\ Hideaki Takagi (Corresponding author) \\ Graduate School of Systems and Information Engineering \\ University of Tsukuba \\ 1-1-1 Tennoudai, Tsukuba-shi, Ibaraki 305-8573, Japan \\ Phone: (029) 853-5003; Fax: (029) 855-3849 \\ e-mail: takagi@sk.tsukuba.ac.jp \\ Sang-Yong Kim \\ Doctoral Program in Systems and Information Engineering \\ University of Tsukuba
}

\begin{abstract}
We propose a new call admission control (CAC) scheme for voice calls in cellular mobile communication networks. It is assumed that the rejection of a hand-off call is less desirable than that of a new call, for a hand-off call loss would cause a severe mental pain to a user. We consider the pains of rejecting new and hand-off calls as different costs. The key idea of our CAC is to restrict the admission of new calls in order to minimize the total expected costs per unit time over the long term. An optimal policy is derived from a semi-Markov decision process in which the intervals between successive decision epochs are exponentially distributed. Based on this optimal policy, we calculate the steady state probability for the number of established voice connections in a cell. We then evaluate the probability of blocking new calls and the probability of forced termination of hand-off calls. In the numerical experiments, it is found that the forced termination probability of hand-off calls is reduced significantly by our CAC scheme at the slight expense of the blocking probability of new calls and the channel utilization.
\end{abstract}

Keywords: mobile communication networks, call admission control, semiMarkov decision process, blocking probability, forced termination, channel utilization. 


\section{Introduction}

Recent years have witnessed a remarkable development in cellular mobile communication techniques. The population of mobile users is explosively growing with the technological and functional progress. Application services provided today are diverse and expanding, including cellular telephony, email of characters and images, and the Internet browsing and transactions. As various traffic will occupy the wireless resources while the frequency bandwidth is limited, the call admission control (CAC) is a mandatory element of mobile communication networks. Traditional policy, i.e., admission if channel is available, which admits a call whenever a channel is available, will not yield priority scheme to various types of traffic. Therefore, some control scheme is needed in order to guarantee the differential quality of service (QoS) requirements as much as possible.

A simple way of giving priority to hand-off calls over new calls is to reserve a certain number of channels exclusively for hand-off calls. Such a guard-channel model was first analyzed by Hong and Rappaport [1], who assumed independent arrivals of new and hand-off calls in a single cell. Their model has been extended to a network of multiple cells in which the hand-off calls for each cell are generated by the outgoing calls from its neighboring cells [2]-[4].

As for the CAC, many schemes have been proposed. A CAC scheme is implemented in [5] by employing a semi-Markov decision process. In [6] the congestion control is carried out for data traffic to ensure that the packet error probability (PEP) of voice be lower than a specified QoS requirement. However, hand-off calls are not considered in these models.

In this paper, we propose a new CAC policy for voice calls by taking into account hand-off calls in cellular networks. We investigate to what degree it has an impact on the traffic performance of the network. Our CAC policy dealing with hand-off calls is executed in the call level. Once the best policy is obtained as a result of the CAC, the hand-off rate is computed from the probabilistic analysis of the behavior of mobile terminals. The steady state probabilities for the number of calls present in the cell are used to calculate the probability of blocking new calls and the probability of forced termination of hand-off calls for voice traffic.

The rest of this paper is organized as follows. Section 2 describes our system model. Section 3 defines performance measures for voice traffic. Section 4 presents numerical results. Concluding remarks are given in Section 5. 


\section{System Model}

In this section, we propose a call level model for a cell in a cellular communication network. We assume that the cellular network is homogeneous such that all cells have the same stochastic properties. We keep track of a sequence of events that each call experiences in the cell. We present a dynamic CAC method using a semi-Markov decision process.

\subsection{Semi-Markov process for the number of calls in a cell}

Let us pay our attention to a single cell. The cell can accommodate calls up to the multiple access capability (MAC) $K$ for voice users, which is determined by a specified QoS requirement. If a new call is placed and the number of existing calls is less than $K$, the call is either admitted into the cell or it is blocked according to the CAC. Similarly, if a hand-off call arrives and the number of existing calls is less than $K$, the hand-off process either succeeds or it is forcibly terminated (i.e., the hand-off process fails), again according to the CAC.

Voice calls have channel holding and cell residence times the lengths of which are a few minutes. The average call holding time and the average cell residence time of a calls are denoted by $1 / \mu_{1}$ and $1 / \mu_{2}$, respectively. Both times are assumed to be exponentially distributed. Therefore, $\mu_{1}$ is the call completion rate, and $\mu_{2}$ is the rate at which a call goes out of the cell. Let $\lambda_{1}$ and $\lambda_{2}$ be the rates at which new and hand-off calls are generated respectively according to independent Poisson processes.

Consider an imbedded Markov chain in which the state is defined by the pair $(k, j)$, where $k$ is the number of calls existing in the cell and $j$ denotes how the state is entered (see below). In this model, we identify a set of Markovian decision epochs such that, if we specify the state at a decision epoch and provide information thereafter, we know the state at the next decision epoch. There are four cases of the state transition from state $(k, j)$ at a decision epoch $(0 \leq k \leq K)$. First, if a new call is placed in a cell and it is admitted into the cell, the chain moves to state $(k+1,1)$ $(0 \leq k \leq K-1)$. Second, if a hand-off call is placed in a cell and it is admitted into the cell, the chain moves to state $(k+1,2)(0 \leq k \leq K-1)$. Third, if a new or hand-off call is placed but rejected, the chain stays in state $(k, j)(0 \leq k \leq K)$. Fourth, if the call is completed or if it leaves the cell as hand-off, the chain moves to state $(k-1,0)(1 \leq k \leq K)$. Note that the last case is a fictitious decision epoch because no decision is made. Figure 1 depicts these transitions in the Markov chain. 


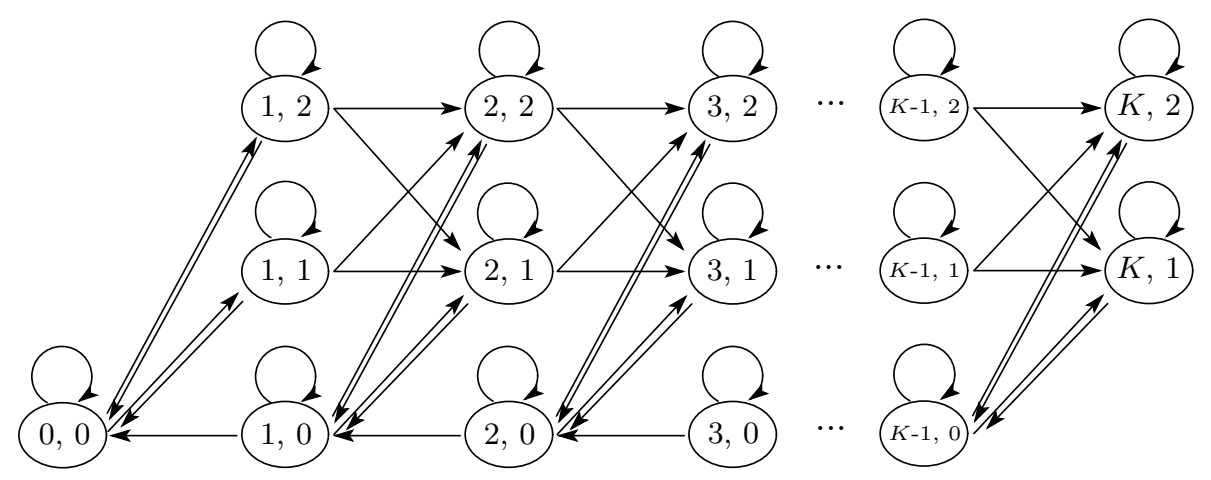

Figure 1: State transition diagram for a Markov chain embedded at decision epochs.

The probabilities of transition from state $(k, j)$ to states $(k-1,0),(k+$ $1,1),(k+1,2)$ and $(k, j)$ are denoted by $q_{k, k-1}, q_{k, k+1}^{(1)}, q_{k, k+1}^{(2)}$, and $q_{k, k}$, respectively, such that $q_{k, k-1}+q_{k, k+1}^{(1)}+q_{k, k+1}^{(2)}+q_{k, k}=1$. They are given in terms of $\mu=\mu_{1}+\mu_{2}, \lambda_{1}, \lambda_{2}$, and the action parameters $a_{(k, 1)}$ and $a_{(k, 2)}$ of the CAC as follows:

$$
\begin{aligned}
q_{k, k-1} & =\frac{k \mu}{\lambda_{1}+\lambda_{2}+k \mu} & & 1 \leq k \leq K \\
q_{k, k+1}^{(1)} & =\frac{\lambda_{1} a_{(k, 1)}}{\lambda_{1}+\lambda_{2}+k \mu} & & 0 \leq k \leq K-1 \\
q_{k, k+1}^{(2)} & =\frac{\lambda_{2} a_{(k, 2)}}{\lambda_{1}+\lambda_{2}+k \mu} & & 0 \leq k \leq K-1 \\
q_{k, k} & =\frac{\lambda_{1}\left(1-a_{(k, 1)}\right)+\lambda_{2}\left(1-a_{(k, 2)}\right)}{\lambda_{1}+\lambda_{2}+k \mu} & & 0 \leq k \leq K,
\end{aligned}
$$

where

$$
\begin{aligned}
& a_{(k, 1)}= \begin{cases}0 & \text { if a new call is rejected } \\
1 & \text { if a new call is admitted }\end{cases} \\
& a_{(k, 2)}= \begin{cases}0 & \text { if a hand-off call is rejected } \\
1 & \text { if a hand-off call is admitted }\end{cases}
\end{aligned}
$$

when $k$ calls are present in the cell $(0 \leq k \leq K-1)$, and $a_{(K, 1)}=a_{(K, 2)}=0$. 
Since $q_{k, k^{\prime}}=q_{k, k^{\prime}}^{(1)}=q_{k, k^{\prime}}^{(2)}=0$ for $\left|k-k^{\prime}\right|>1$, the Markov chain is a birthand-death process. Assuming that the chain is ergodic, let $p_{k, j} ; 0 \leq k \leq K$, $0 \leq j \leq 2$, be the steady state probability for the chain to be in state $(k, j)$. Then we have the set of balance equations:

$$
p_{k, j}=\left\{\begin{array}{ll}
\left(\sum_{i=0}^{2} p_{k+1, i}\right) \cdot q_{k+1, k}+p_{k, 0} \cdot q_{k, k} & j=0 \\
\left(\sum_{i=0}^{2} p_{k-1, i}\right) \cdot q_{k-1, k}^{(1)}+p_{k, 1} \cdot q_{k, k} & j=1 \\
\left(\sum_{i=0}^{2} p_{k-1, i}\right) \cdot q_{k-1, k}^{(2)}+p_{k, 2} \cdot q_{k, k} & j=2
\end{array},\right.
$$

and the normalization condition:

$$
\sum_{k=0}^{K} \sum_{j=0}^{2} p_{k, j}=1 .
$$

Let $\widetilde{p}_{k, j}$ be the steady state probability of state $(k, j)$ at an arbitrary point in the continuous-time domain. Then, from the theory of semi-Markov processes [7, Section 9-1], we have

$$
\widetilde{p}_{k, j}=\frac{p_{k, j} \cdot \eta_{k}}{\eta} \quad 0 \leq k \leq K, 0 \leq j \leq 2,
$$

where

$$
\eta_{k}=\frac{1}{\lambda_{1}+\lambda_{2}+k \mu}
$$

is the mean sojourn time in state $(k, j)$, and $\eta$ is the average time interval between the successive points of state transitions, given by

$$
\eta=\sum_{k=0}^{K} \sum_{j=0}^{2} p_{k, j} \cdot \eta_{k}
$$

\subsection{Call admission control policy}

Accidents that ongoing conversations are forcibly terminated generally displease the user more than initial access failures. Thus it makes sense that we weight different rejection costs for new calls and hand-off calls and that 
we try to minimize the average cost per unit time over the long term. The cost should quantify the strength of stress that a rejected user feels.

A policy that rejects a new call even if there are available channels may reject fewer hand-off calls on average than another policy that accepts a new call whenever there are available channels. Here, the "policy" means an action to take in every state. Finding the optimal policy is the goal of our CAC policy. In other words, the optimal choice of actions (accept or reject a call) is made in each state to minimize the average cost per unit time over the long term.

In order to find the optimal policy, we employ a semi-Markov decision process as in [5]. The process is observed when a conversation is completed, a calling user goes out of the cell, a new call is placed, or a hand-off call arrives. If we assume that each of these events occurs in a Poisson process, the intervals between two successive observation points, called decision epochs, are exponentially distributed. After observing the process, a decision is made according to the policy and the corresponding cost is incurred as a consequence of the decision made. The set of possible states in the process is denoted by

$$
\mathcal{I}=\{\boldsymbol{x}=(k, j) \mid 0 \leq k \leq K, 0 \leq j \leq 2\} \backslash\{\boldsymbol{x}=(K, 0)\},
$$

where state $\boldsymbol{x}=(k, j)$ is defined in Section 2.1.

Suppose that the cost function is given by

$$
C_{\boldsymbol{x}}\left(\boldsymbol{a}_{k}\right)=\left(1-\boldsymbol{a}_{k}\right) \gamma_{j} \quad \boldsymbol{x}=(k, j),
$$

where $\boldsymbol{a}_{k}=\left(a_{(k, 1)}, a_{(k, 2)}\right)$ is the action pair. $C \boldsymbol{x}\left(\boldsymbol{a}_{k}\right)$ represents the immediate cost incurred until the next decision epoch if action $\boldsymbol{a}_{k}$ is chosen at state $\boldsymbol{x}=(k, j) \cdot \gamma_{j}$ quantifies the strength of stress which a rejected user feels at state $\boldsymbol{x}=(k, j)$, where we let $\gamma_{0}=0$.

The expected time until the next decision epoch in state $\boldsymbol{x}$ is given by

$$
\tau \boldsymbol{x}=\frac{1}{\lambda_{1}+\lambda_{2}+k \mu} \quad \boldsymbol{x}=(k, j) .
$$

The transition probability from state $\boldsymbol{x}=(k, j)$ to state $\boldsymbol{x}^{\prime}$ if action $\boldsymbol{a}_{k}$ is chosen at state $\boldsymbol{x}$ is given by 


$$
P_{\boldsymbol{x}, \boldsymbol{x}^{\prime}}\left(\boldsymbol{a}_{k}\right)=\left\{\begin{array}{cl}
q_{k, k-1} & \boldsymbol{x}^{\prime}=(k-1,0) \\
q_{k, k+1}^{(1)} & \boldsymbol{x}^{\prime}=(k+1,1) \\
q_{k, k+1}^{(2)} & \boldsymbol{x}^{\prime}=(k+1,2) \\
q_{k, k} & \boldsymbol{x}^{\prime}=\boldsymbol{x} \\
0 & \text { otherwise }
\end{array}\right.
$$

Now, the value-iteration algorithm of a semi-Markov decision process is applied to our CAC in order to determine the optimal policy. To do so, we convert the semi-Markov decision model into a discrete-time Markov decision model such that the average costs per unit time over the long term of each stationary policy are the same in both models. This is done by a method called uniformization [8, Section 3.4].

For the conversion, we choose a time unit $\tau$ in the discrete-time model such that

$$
0 \leq \tau \leq \min _{\boldsymbol{x} \in \mathcal{I}} \tau \boldsymbol{x}=\frac{1}{\lambda_{1}+\lambda_{2}+K \mu}
$$

Then a decision epoch occurs in time $\tau$ with probability $\tau / \tau_{\boldsymbol{x}}$ and it does not with probability $1-\tau / \tau \boldsymbol{x}$. The transition probabilities in the discrete-time model are given by

$$
\bar{P}_{\boldsymbol{x}, \boldsymbol{x}^{\prime}}\left(\boldsymbol{a}_{k}\right)=\left\{\begin{array}{ll}
\frac{\tau}{\tau_{\boldsymbol{x}}} P_{\boldsymbol{x}, \boldsymbol{x}^{\prime}}\left(\boldsymbol{a}_{k}\right) & \boldsymbol{x}^{\prime} \neq \boldsymbol{x}=(k, j) \\
\frac{\tau}{\tau_{\boldsymbol{x}}} P_{\boldsymbol{x}, \boldsymbol{x}^{\prime}}\left(\boldsymbol{a}_{k}\right)+\left\{1-\frac{\tau}{\tau_{\boldsymbol{x}}}\right\} & \boldsymbol{x}^{\prime}=\boldsymbol{x}=(k, j)
\end{array} .\right.
$$

If the current state is $\boldsymbol{x}=(k, j)$ and action $\boldsymbol{a}_{k}$ is chosen, the average cost per unit time until the next decision epoch is given by $C_{\boldsymbol{x}}\left(\boldsymbol{a}_{k}\right) / \tau_{\boldsymbol{x}}$. The quantity $V_{n}(\boldsymbol{x})$ is introduced as the minimal total expected cost with $n$ steps left to the time horizon when the current state is $\boldsymbol{x}$ in the discrete-time process. Since the goal is to minimize the average cost per unit time over the long term, we must go backward in the time axis until the one-step difference $V_{n}(\boldsymbol{x})-V_{n-1}(\boldsymbol{x})$ converges to the minimal average cost per unit time.

The value-iteration algorithm to find the optimal policy in the discretetime model is described as follows:

1. Choose $V_{0}(\boldsymbol{x})$ such that $0 \leq V_{0}(\boldsymbol{x}) \leq \frac{1}{\tau_{\boldsymbol{x}}} \min _{\boldsymbol{a}_{k}}\left\{C_{\boldsymbol{x}}\left(\boldsymbol{a}_{k}\right)\right\}$ for all $\boldsymbol{x}=$ $(k, j) \in \mathcal{I}$. Set $n:=1$. 
2. Compute for $\boldsymbol{x}=(k, j) \in \mathcal{I}$

$$
V_{n}(\boldsymbol{x})=\min _{\boldsymbol{a}_{k}}\left[\frac{C_{\boldsymbol{x}}\left(\boldsymbol{a}_{k}\right)}{\tau_{\boldsymbol{x}}}+\frac{\tau}{\tau_{\boldsymbol{x}}} \sum_{\boldsymbol{x}^{\prime} \in \mathcal{I}} P_{\boldsymbol{x}, \boldsymbol{x}^{\prime}}\left(\boldsymbol{a}_{k}\right) V_{n-1}\left(\boldsymbol{x}^{\prime}\right)+\left\{1-\frac{\tau}{\tau_{\boldsymbol{x}}}\right\} V_{n-1}(\boldsymbol{x})\right]
$$

and determine the action $\boldsymbol{a}_{k}$ that minimizes the right-hand side of (15).

3. Compute the upper and lower bounds of the one-step difference by

$$
M_{n}=\max _{\boldsymbol{x} \in \mathcal{I}}\left\{V_{n}(\boldsymbol{x})-V_{n-1}(\boldsymbol{x})\right\} \quad \text { and } \quad m_{n}=\min _{\boldsymbol{x} \in \mathcal{I}}\left\{V_{n}(\boldsymbol{x})-V_{n-1}(\boldsymbol{x})\right\}
$$

4. If $0 \leq M_{n}-m_{n} \leq \epsilon m_{n}$, then stop the algorithm with the optimal set of actions for all $\boldsymbol{x}$. Otherwise $n:=n+1$ and go to step 2 .

Here, $\epsilon$ is a prespecified small positive constant (tolerance number) for stopping the iteration. In our numerical experiments, the algorithm had convergence with $\epsilon=10^{-3}$.

Once the optimal policy $\left\{\boldsymbol{a}_{k}=\left(a_{(k, 1)}, a_{(k, 2)}\right) ; 0 \leq k \leq K-1\right\}$ is determined, we can obtain the steady state probabilities $p_{k, j}$ and $\widetilde{p}_{k, j} ; 0 \leq k \leq$ $K, 0 \leq j \leq 2$, as shown in Section 2.1 .

\section{Performance Measures}

In this section, we introduce some performance measures in order to compare the CAC model with various ratios $\gamma_{2} / \gamma_{1}$ and the non-CAC model. Namely, we will use the probability of blocking new calls and the probability of forced termination of hand-off calls. We also consider the channel utilization as a measure of effective use of the channels.

\subsection{Blocking and forced termination probabilities}

There are two situations which irritate users. One is that users who newly try to call someone are rejected by the system due to no available channel (i.e., blocking new calls). The other is that users who are on the phone and crossing the cell boundary are forcibly broken off (i.e., forced termination of hand-off calls). Therefore, we consider the probability of blocking new calls and the probability of forced termination of hand-off calls as performance measures from the user's viewpoint. 
If a new call is placed in a cell and the action pair that follows the CAC policy is "reject", it results in the blocking of the new call. If a hand-off call arrives in a cell and it is rejected according to the CAC policy, it means the forced termination of the hand-off call. It is assumed that new calls and hand-off calls arrive according to Poisson processes independently. Let $P_{b}$ represent the probability of blocking new calls and let $P_{f}$ be the probability of forced termination of hand-off calls. Using the PASTA (Poisson Arrivals See Time Averages) property [7, Section 11-2], we have

$$
\begin{aligned}
& P_{b}=\sum_{a_{(k, 1)}=0} \sum_{j=0}^{2} \widetilde{p}_{k, j} \\
& P_{f}=\sum_{a_{(k, 2)}=0} \sum_{j=0}^{2} \widetilde{p}_{k, j},
\end{aligned}
$$

where $\widetilde{p}_{k, j} ; 0 \leq k \leq K, 0 \leq j \leq 2$ is the steady state probability for the number of calls in a cell at an arbitrary time, given in equation (7).

\subsection{Channel utilization}

Another performance yardstick is the channel utilization. Channel capacity is the maximum number of available channels, that is, the multiple access capability $K$, stated in Section 2.1. Let $N$ be the average number of channels in use at an arbitrary time. It is given by

$$
N=\sum_{k=0}^{K} \sum_{j=0}^{2} k \cdot \widetilde{p}_{k, j} .
$$

Then the channel utilization $U$ is given as the ratio of the average number of channels in use to the channel capacity as

$$
U=\frac{N}{K}
$$

It is obvious that $U=1$ is an ideal situation from the system operator's viewpoint of channel usage.

\section{Numerical Experiments}

Numerical experiments have been carried out in order to evaluate to what degree our CAC scheme has an impact on the performance of voice traffic. It 
has been assumed throughout our experiments that the average call holding time is $1 / \mu_{1}=1.5$ (minutes) and that the average cell residence time is $1 / \mu_{2}=5$ (minutes). The number of channels is assumed to be $K=22$; see Appendix for this choice.

We examine the effectiveness of the CAC by means of the blocking and forced termination probabilities for the new and hand-off calls, respectively, as well as the channel utilization. In the legend of the figures shown below, the "non-CAC" means the result when the optimization of action is not made. For the CAC cases, "ratio $=1$ " means that the cost of rejecting new and hand-off calls is the same $\left(\gamma_{2} / \gamma_{1}=1\right)$, "ratio $=10 "$ and "ratio $=100$ " mean that the rejection of hand-off calls gives users 10 and 100 times more stress (cost) over new calls $\left(\gamma_{2} / \gamma_{1}=10\right.$ and 100), respectively.

\subsection{Calculation of the hand-off call arrival rate}

Suppose that the network consists of independent and statistically identical cells. Then the inbound hand-off rate can be considered equal to the outbound rate to adjacent cells. Given the steady state probabilities for the number of simultaneous calls in the cell, the inbound hand-off rate $\lambda_{2}$ from neighboring cells can be determined by the fixed-point method $[2,3]$. By this method, the algorithm to calculate the rate $\lambda_{2}$ is given as follows:

1. Initialize $\lambda_{2}=0$.

2. Calculate $\widetilde{p}_{k, j} ; 0 \leq k \leq K, 0 \leq j \leq 2$ as shown in Section 2 .

3. Compute

$$
\lambda_{2}=\sum_{k=0}^{K} \sum_{j=0}^{2}\left(k \mu_{2}\right) \cdot \widetilde{p}_{k, j} .
$$

4. Repeat steps 2 and 3 until $\lambda_{2}$ converges.

We note that the value-iteration, stated in Section 2.1, is conducted during step 2 with the interim value of $\lambda_{2}$ to get the optimal policy $\boldsymbol{a}_{k}$ for the CAC model. In our experiments, we have used

$$
\tau=\frac{1}{\lambda_{1}+\lambda_{2}+2 K \mu}
$$

for the value-iteration algorithm.

With the value of hand-off rate $\lambda_{2}$ that has converged, we have the relation 


$$
\lambda_{2}=K U \mu_{2},
$$

which is reasonable as $K U=N$ is the average number of channels being used at an arbitrary time.

We omit plotting $\lambda_{2}$ as a function of $\lambda_{1}$ as $\lambda_{2}$ is proportional to the channel utilization $U$ shown in Figure 5 below. From Figure 5 we can say that the hand-off rate $\lambda_{2}$ is less for the CAC model with larger ratio $\gamma_{2} / \gamma_{1}$, because new call arrivals are more often blocked then in order to reserve more channels for accepting hand-off calls.

\subsection{Performance results and discussion}

Before discussing the performance measures, let us look at the optimized action at decision epochs. Our optimization has resulted in $a_{(k, 2)}=1$ for $0 \leq k \leq K-1$, i.e., hand-off calls are always admitted as far as channels are available. On the other hand, there exists an upper bound for the number of available channels over which new calls are blocked in the CAC model. Such bound depends on the ratio $\gamma_{2} / \gamma_{1}$.

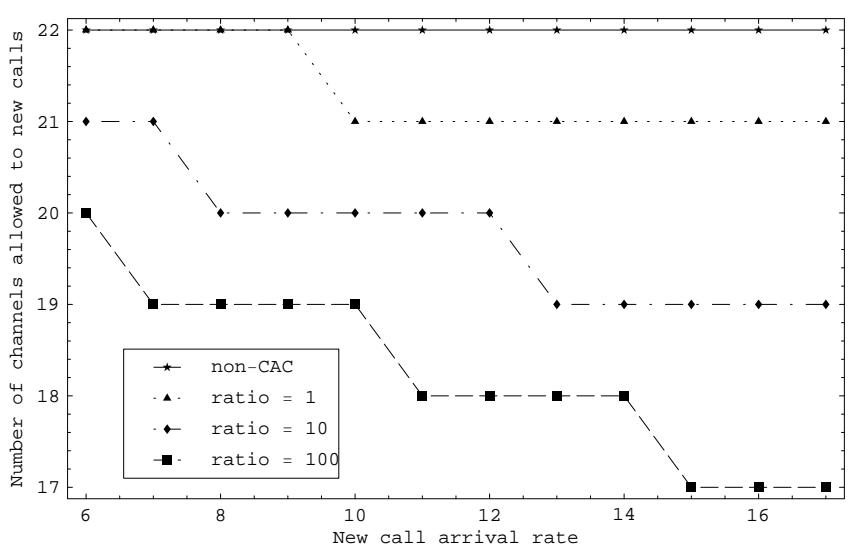

Figure 2: Number of channels allowed to new calls.

Figure 2 shows that the maximum number of channels, $k_{\max }$, allowed to new calls as a result of optimization, where

$$
k_{\max }=\max _{0 \leq k \leq K}\left\{k \mid a_{(k, 1)}=1\right\} .
$$


For the CAC models with given ratios $\gamma_{2} / \gamma_{1}, k_{\max }$ decreases monotonously as a function of $\lambda_{1}$. This implies that fewer channels can be used by new calls as the traffic increases. For a given level of $\lambda_{1}, k_{\max }$ is less for larger $\gamma_{2} / \gamma_{1}$, which represents the effects of our CAC.

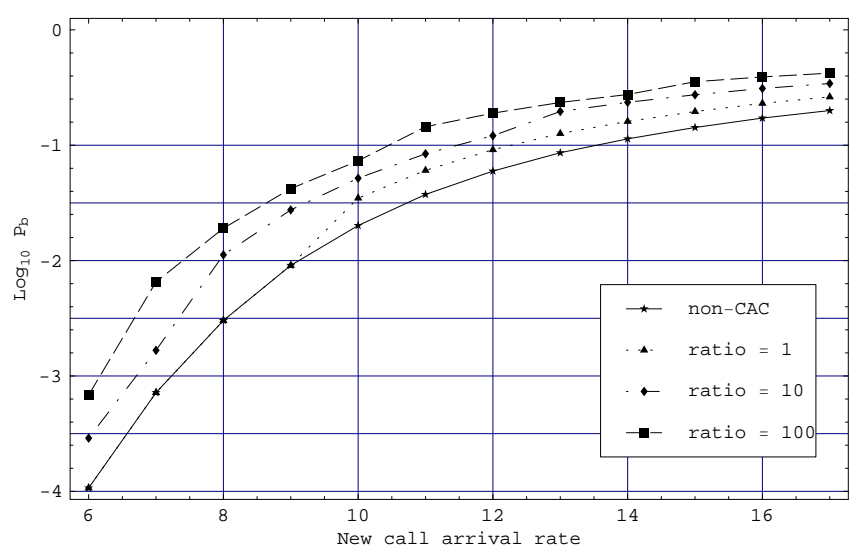

Figure 3: Blocking probability of new calls.

Figure 3 shows the blocking probability $P_{b}$ of new calls as a function of the new call arrival rate $\lambda_{1}$. Since our CAC restricts new calls for the benefit of hand-off calls, it is reasonable that the larger the ratio $\gamma_{2} / \gamma_{1}$, the larger the blocking probability. For example, the blocking probabilities after implementation of the CAC with $\gamma_{2} / \gamma_{1}=10$ and $\gamma_{2} / \gamma_{1}=100$ are 2.7 and 6.4 times larger, respectively, than that under the non-CAC at $\lambda_{1}=6$.

In Figure 4, we plot the forced termination probability $P_{f}$ of hand-off calls as a function of the new call arrival rate $\lambda_{1}$. As hand-off calls have great advantage over new calls under the $\mathrm{CAC}$ with large ratios, the forced termination probabilities are smaller under the CAC with larger ratio. Let us note that the forced termination probability drops at $\lambda_{1}=10$ for $\gamma_{2} / \gamma_{1}=1$, $\lambda_{1}=8$ and 13 for $\gamma_{2} / \gamma_{1}=10$, and $\lambda_{1}=11$ and 15 for $\gamma_{2} / \gamma_{1}=100$. The reason is that the number of available channels under which the optimal policy rejects new calls is decremented by one at those arrival rates as shown in Figure 2. However the forced termination probability increases at $\lambda_{1}=7$ for $\gamma_{2} / \gamma_{1}=100$, although the optimal policy starts rejecting new calls here too. The reason may be that the increasing rate of hand-off calls outperforms the effect of rejecting new calls.

We should not overlook the difference in the effects of CAC at low and 




Figure 4: Forced termination probability of hand-off calls.

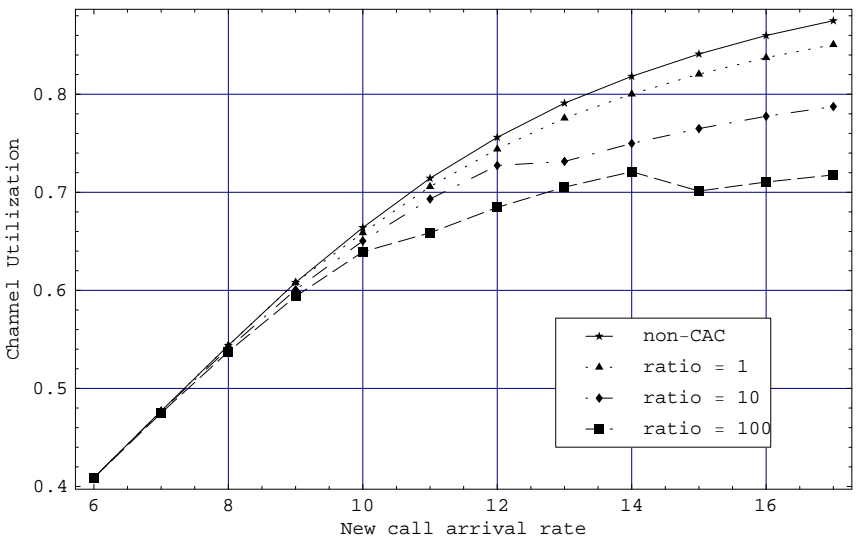

Figure 5: Channel utilization. 
high load conditions. Because the blocking probabilities whether under the $\mathrm{CAC}$ or non-CAC are very large at heavy load, the effect of CAC at heavy load is relatively small compared with that at light load in Figure 3. On the other hand, the effect of CAC on the forced termination probabilities at heavy load is larger than that at light load in Figure 4.

Figure 5 shows the channel utilization. Channel utilization values under the CAC with $\gamma_{2} / \gamma_{1}=1,10$, and 100 is $97.4,90.4$, and $82.6 \%$, respectively, of the value under the non-CAC at $\lambda_{1}=16$. Thus channels are slightly less utilized under the $\mathrm{CAC}$ than under the non-CAC. This is a negative effect of $\mathrm{CAC}$ on the channel utilization, but it leads to the great reduction in the probability of forced termination of hand-off calls as shown in Figure 4.

\section{Concluding Remarks}

In this paper, we have proposed a CAC scheme for voice traffic and analyzed its effects for cellular mobile communication networks. Our problem has been modeled by a two-dimensional Markov chain, a semi-Markov process, and a (semi-)Markov decision process. According to the numerical experiments, our CAC reduces significantly the forced termination probability of hand-off calls, while it marginally increases the blocking probability of new calls and suppresses the channel utilization.

As usual with optimization by (semi-)Markov decision processes, the computational time may prohibit the application of our CAC to the dynamic control of real systems. However, our results could be used as a benchmark against those by quick approximate optimizing methods.

\section{References}

[1] D. H. Hong and S. S. Rappaport, "Traffic model and performance analysis for cellular mobile radio telephone systems with prioritized and nonprioritized handoff procedures," IEEE Transactions on Vehicular Technology, Vol. VT-35, No.3, pp. 77-92, August 1986.

[2] K. Sakamaki and H. Takagi, "Evaluation of call loss and forced termination probabilities in cellular communication systems," The Transactions of the Institute of Electronics, Information and Communication Engineers B-II, Vol. J80-B-II, No.3, pp. 231-238, March 1997 (in Japanese). 
[3] H. Takagi, K. Sakamaki, and T. Miyashiro, "Call loss and forced termination probabilities in cellular radio communication networks with nonuniform traffic conditions," IEICE Transactions on Communications, Vol. E82-B, No.9, pp. 1496-1504, September 1999.

[4] M. Ohmikawa and H. Takagi, "Call loss probabilities in CDMA cellular mobile communication networks," The Transactions of the Institute of Electronics, Information and Communication Engineers B, Vol. J82-B, No.12, pp. 2311-2319, December 1999 (in Japanese). English translation in Electronics and Communications in Japan, Part 2: Electronics, Vol. 84, No.1, pp. 11-20, 2001, Scripta Technica.

[5] W-B Yang and E. Geraniotis, "Admission policies for integrated voice and data traffic in CDMA packet radio networks," IEEE Journal on Selected Areas in Communications, Vol. 12, No.4, pp. 654-664, May 1994.

[6] T. Liu and J. A. Silverster, "Joint admission/congestion control for wireless CDMA systems supporting integrated services," IEEE Journal on Selected Areas in Communications, Vol. 16, No.6, pp. 845-857, August 1998.

[7] D. P. Heymann and M. J. Sobel, Stochastic Models in Operations Research, Volume I: Stochastic Processes and Operating Characteristics. McGraw-Hill Book Company, New York, 1982.

[8] H. C. Tijms, Stochastic Models: An Algorithmic Approach. John Wiley \& Sons, Chichester, England, 1994.

[9] J. S. Lehnert and M. B. Pursley, "Error probabilities for binary directsequence spread spectrum communications with random signature sequences," IEEE Transactions on Communications, Vol. COM-35, No.1, pp. 87-98, January 1987.

[10] R. K. Morrow, Jr. and J. S. Lehnert, "Bit-to-bit error dependence in slotted DS/SSMA packet systems with random signature sequences," IEEE Transactions on Communications, Vol. 37, No.10, pp. 1052-1061, October 1989.

[11] J. M. Holtzman, "A simple, accurate method to calculate spreadspectrum multiple-access error probabilities," IEEE Transactions on Communications, Vol. 40, No.3, pp. 461-464, March 1992. 
[12] R. K. Morrow, Jr., "Accurate CDMA BER calculations with low computational complexity," IEEE Transactions on Communications, Vol. 46, No.11, pp. 1413-1417, November 1998.

[13] T. Ojanperä and R. Prasad, ed., Wideband CDMA for Third Generation Mobile Communications. Artech House, Norwood, Massachusetts, 1998.

\section{Appendix: Multiple Access Capability}

This appendix presents an approximate method for evaluating the error probability of a voice packet as a function of the multiple access capability (MAC) $K$, the maximum number of simultaneous users. This is used to choose the value of $K$ in our numerical experiments (given in Section 4) when the packet error probability (PEP) is the QoS requirement.

The direct-sequence code division multiple access (DS-CDMA) is considered as a multiple access technique. It is an averaging system which reduces the interference by averaging the signal power over a long time interval. Therefore, the more multiple DS signals overlap in time and frequency the noisier the received signal is, resulting in the degradation of QoS. To guarantee various QoS requirements, we must adjust the MAC values for each type of users. For the voice traffic, we consider only the PEP of the voice packet as a specified QoS requirement.

Various approximation techniques for the bit error probability (BEP) have been developed for the DS-CDMA radio system using binary phase shift keyed (BPSK) signaling. In [9], expressions are developed for the multiple access interference (MAI) and the upper and lower bounds are given on the average error probability for a direct-sequence spread spectrum multiple access (DS-SSMA) system. An accurate approximation, called "improved Gaussian approximation," is subsequently presented in [10]. Another approximation that is simpler but maintains the same accuracy is proposed in [11], which is further simplified in [12]. Here, we employ an approximation given in [12], which simplifies the improved Gaussian approximation while maintaining the same accuracy.

It is assumed that interfering signal sequences are random and that MAI is the only source of bit errors. Suppose that the ratio $N=T_{b} / T_{c}$ is a constant, where $T_{b}$ is the duration of each encoded data bit and $T_{c}$ is the duration of each chip in the signal sequence, and that the system has $K$ simultaneous users. It is well known that the BEP can be approximately calculated by finding the average signal-to-noise ratio (SNR) $x$ and using the $Q$ function 


$$
Q(x)=\frac{1}{\sqrt{2 \pi}} \int_{x}^{\infty} \exp \left(-\frac{u^{2}}{2}\right) d u .
$$

According to [12], the simplified improved Gaussian approximation yields results close to the improved Gaussian approximation as long as the BEP is larger than about $10^{-6}$. If the mean $\mu$ and standard deviation $\sigma$ of the distribution of the MAI are available, the BEP is given by

where

$$
\operatorname{BEP}(K)=\frac{2}{3} a+\frac{1}{6} b+\frac{1}{6} c,
$$

$$
a=Q\left(\sqrt{\frac{N^{2}}{\mu}}\right) ; b=Q\left(\sqrt{\frac{N^{2}}{\mu+\sqrt{3} \sigma}}\right) ; c=Q\left(\sqrt{\frac{N^{2}}{\mu-\sqrt{3} \sigma}}\right) .
$$

The values for $\mu$ and $\sigma^{2}$ are given by

$$
\mu=(K-1) \frac{N}{3} ; \quad \sigma^{2} \approx(K-1) \frac{23 N^{2}}{360} .
$$

Let us now consider the PEP using the above BEP. We can express the PEP in a closed form if we ignore the bit-by-bit error dependency for ease of computation. Suppose that one packet length is $L$ bits and the block error correction capability is incorporated into the data packet which can correct $t$ or fewer bits. Then the PEP is given by

$$
\operatorname{PEP}(K)=1-\sum_{i=0}^{t}\left(\begin{array}{c}
L \\
i
\end{array}\right) \operatorname{BEP}(K)^{i}(1-\operatorname{BEP}(K))^{L-i}
$$

This equation yields the MAC, $K$, for voice call users so that $\operatorname{PEP}(K)$ satisfies the QoS requirement $\operatorname{PEP}(K)<10^{-2}$.

The IS-95 air interface [13] says that the signal at a rate of $19.2 \mathrm{Kbps}$ is spread with an orthogonal Walsh code at a rate of $1.2288 \mathrm{Mcps}$, that is, $N=1228.8 / 19.2=64$. Data packets to be transmitted are first grouped into 20 millisecond frames. If exactly one packet is transmitted in a frame and the bit rate is $14.4 \mathrm{Kbps}$, the packet length $L$ is $14.4 \times 20=288$ bits. Furthermore, it is assumed that each packet has the block error correction capability that can correct two or fewer bit errors $(t=2)$.

We have discussed that $K$ is dependent on system parameters and the specified QoS requirement. The system has to ensure that the PEP of transmitted data packets never violates the specified QoS requirement. Referring to Figure 6, obtained by equation (A.5), we find $K=22$ as the number of simultaneous users such that the PEP is about $10^{-2}$. 




Figure 6: PEP versus MAC. 